\title{
Fiducial Marker
}

\author{
Osamu $T^{* 1}$ and Yoshikatsu Yoshinaga $\mathbf{Y}^{1}$ \\ Department of Radiation Oncology, Asahi University Hospital
}

"Corresponding author: Osamu Tanaka, Department of Radiation Oncology, Asahi University Hospital, 2-23 Hashimoto-cho, Gifu city, Gifu, 500-8523, Japan. Tel: +81-58-253-8001; Fax: +81-58-253-8002; E-mail: c.bluered@gmail. com

\section{Editorial \\ Placement of Fiducial Markers}

Placement of fiducial markers uses imaging guidance to place small metal objects called fiducial markers in or near the tumor in preparation for radiation therapy. The markers allow for more accurate localization of the tumor and allow the treatment team to deliver the maximum radiation dose to the tumor. Preserves healthy tissue [1].

Your doctor will tell you how to prepare, including changing your medication schedule. If you may be pregnant, contact your doctor to discuss your recent illness, medical condition, allergies, or medications such as aspirin. You may be advised to stop taking aspirin, nonsteroidal anti-inflammatory drugs (NSAIDs), or anticoagulants a few days before treatment. You may also be told not to eat or drink anything a few hours before the procedure. Please wear loose and comfortable clothes. You may be required to wear a gown [2].

\section{What is the Placement of Fiducial Markers?}

Placement of fiducial markers is an image-guided procedure often performed by interventional radiologists in preparation for certain types of radiation therapy, such as stereotactic radiotherapy (SRS) or stereotactic radiotherapy (SBRT), or proton therapy [3]. The fiducial markers are small metal (usually gold) spheres, coils, or cylinders that are the same size as the rice grains that are placed in or near the tumor to guide the placement of the radiation beam during treatment.

\section{What are the General Uses of the Procedure?}

Fiducial markers define the lesions within the following soft tissues and are implanted to target them.

- Chest, including lungs and chest wall

- Abdomen including liver, gallbladder, kidneys, pancreas

- Pelvis including prostate

- Head and neck.

\section{How do I Prepare?}

Prior to your procedure, your blood may be tested to determine how well your kidneys are working, and whether your blood is coagulating normally. All medications taken, including supplements, should be reported to your doctor. If you are allergic, please report local anesthetics, general anesthesia, or contrast agents containing iodine, among others [4]. Your doctor may advise you to stop taking aspirin, nonsteroidal anti-inflammatory drugs (NSAIDs), or anticoagulants before the procedure for a specified period of time. Also, inform your doctor about recent illnesses and other medical conditions. You should always inform your doctor and x-ray technician if your woman may be pregnant. Many imaging tests are not done during pregnancy to avoid exposing the fetus to radiation. If you need $\mathrm{x}$-rays, take precautions [5].

You will receive specific instructions on how to prepare, including any changes you may need to make to your regular dosing schedule. You may be told not to eat or drink anything a few hours before the procedure, except for medication. You may be required to remove some or all of your clothes and wear a gown during the exam. glasses, metal objects and clothing that can interfere with X-ray images may also be removed. After your procedure, you should plan to have your relatives or friends drive you home [6].

\section{What Does the Device Look Like?}

The fiducial marker is embedded using a delivery device similar to a biopsy needle. The placement of fiducial markers is guided by computed tomography (CT) or ultrasound and may also involve the use of an endoscope, an illuminated optical instrument [7].

\section{How does the Procedure Work?}

The fiducial markers are placed using a needle inserted into the area of the lesion with the help of computed tomography (CT) or ultrasound (US). Next, the pre-filled marker on the needle is moved to the carefully marked position tumor.

\section{How are the Steps Performed?}

Placement of fiducial markers is usually performed as an outpatient procedure (several days) before the treatment plan begins.

Imaging may occur before the procedure begins.

You may be connected to a monitor that tracks your heart rate, blood pressure, oxygen level, and pulse. 


\section{Conflicts of Interest: \\ No}

\section{Financial Support:}

No

\section{References}

1. Slagowski JM, Colbert LE, Cazacu IM, Singh BS, Martin R, Koay EJ, et al. Evaluation of the Visibility and Artifacts of 11 Common Fiducial Markers for Image Guided Stereotactic Body Radiation Therapy in the Abdomen. Pract Radiat Oncol. 2020:S1879-8500(20)30008-4. doi: 10.1016/j.prro.2020.01.007. Online ahead of print. PMID: 31988039

2. van den Ende RPJ, Rigter LS, Kerkhof EM, van Persijn van Meerten EL, Rijkmans EC, Lambregts DMJ, et al. MRI visibility of gold fiducial markers for imageguided radiotherapy of rectal cancer. Radiother Oncol. 2019;132:93-99. doi: 10.1016/j.radonc.2018.11.016. Epub 2018 Dec 21. PMID: 30825976.

3. Wegener D, Zips D, Thorwarth D, Weiß J, Othman AE, Grosse U, et al. Precision of T2 TSE MRI-CT-image fusions based on gold fiducials and repetitive T2 TSE MRIMRI-fusions for adaptive IGRT of prostate cancer by using phantom and patient data. Acta Oncol. 2019;58(1):88-94. doi: 10.1080/0284186X.2018.1518594. Epub 2018 Sep 28. PMID: 30264629.
4. Suzuki T, Saito M, Onishi H, Mochizuki K, Satani K, Yamazaki A, et al. Comparison of CT artifacts and image recognition of various fiducial markers including two types of thinner fiducial markers for CyberKnife treatment. Rep Pract Oncol Radiother. 2020;25(1):117-124. doi: 10.1016/j.rpor.2019.12.005. Epub 2019 Dec 9. PMID: 31908605.

5. Greer P, Martin J, Sidhom M, Hunter P, Pichler P, Choi $\mathrm{JH}$, et al. A Multi-center Prospective Study for Implementation of an MRI-Only Prostate Treatment Planning Workflow. Front Oncol. 2019;29;9:826. doi: 10.3389/ fonc.2019.00826. eCollection 2019.PMID: 31555587. Free PMC article.

6. Kubota Y, Katoh H, Shibuya K, Shiba S, Abe S, Sakai M, et al. Comparison between bone matching and marker matching for evaluation of intra- and inter-fractional changes in accumulated dose of carbon ion radiotherapy for hepatocellular carcinoma. Radiother Oncol. 2019;137:7782. doi: 10.1016/j.radonc.2019.04.026. Epub 2019 May 9.PMID: 31078014 .

7. Napieralska A, Majewski W, Kulik R, Głowacki G, Miszczyk L. A comparison of treatment outcome between fiducial-based and bone-based image guided radiotherapy in prostate cancer patients. Radiat Oncol. 2018;27:13(1):235. doi: 10.1186/s13014-018-1171-2.PMID: 30482212. Free PMC article. 